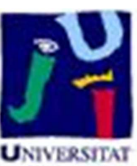

Título artículo / Títol article: How deeply do we include robotic agents in the self?

Autores / Autors

Anna Stenzel, Eris Chinellato, Ángel Pascual del Pobil y Ferré, Markus Lappe, Roman Liepelt

Revista:

International Journal of Humanoid Robotics

Versión / Versió:

Postprint de l'autor

Cita bibliográfica / Cita bibliogràfica (ISO 690):

STENZEL, Anna, et al. How Deeply do we include robotic Agents in the Self?.International Journal of Humanoid Robotics, 2013, vol. 10, no 01.

url Repositori UJI: 


\title{
HOW DEEPLY DO WE INCLUDE ROBOTIC AGENTS IN THE
} SELF?

\author{
ANNA STENZEL \\ University of Münster, Institute for Psychology \\ Fliednerstr. 21, D-48149 Muenster, Germany \\ anna.stenzel@uni-muenster.de \\ ERIS CHINELLATO \\ Imperial College London, Department of Electrical and Electronic Engineering \\ South Kensington Campus, London SW7 2AZ, United Kingdom \\ e.chinellato@imperial.ac.uk \\ ANGEL P. DEL POBIL \\ Jaume I University, Robotic Intelligence Laboratory \\ Avda. Vicente Sos Baynat s/n, E-12071, Castellon de la Plana, Spain \\ Sungkyunkwan University, Department of Interaction Science \\ B307 International Hall, 53 Myeongnyun-dong 3-ga, Jongno-gu, Seoul 110-745, South Korea \\ pobil@icc.uji.es \\ MARKUS LAPPE \\ University of Münster, Institute for Psychology \\ Fliednerstr. 21, D-48149 Muenster, Germany \\ mlappe@uni-muenster.de \\ ROMAN LIEPELT \\ University of Münster, Institute for Psychology \\ Fliednerstr. 21, D-48149 Muenster, Germany \\ roman.liepelt@uni-muenster.de \\ Received Day Month Year \\ Revised Day Month Year \\ Accepted Day Month Year
}

In human-human interactions a consciously perceived high degree of self-other overlap is associated with a higher degree of integration of the other person's actions into one's own cognitive representations. Here, we report data suggesting that this pattern does not hold for human-robot interactions. Participants performed a social Simon Task with a robot, and afterwards indicated the degree of self-other overlap with the help of the Inclusion of the Other in the Self (IOS) scale. We found no overall correlation between the social Simon Effect (as an indirect measure of self-other overlap) and the IOS score (as a direct measure of self-other overlap). For female participants we even observed a negative correlation. Our findings suggest that conscious and unconscious evaluations of a robot may come to different results, and hence point to the importance of carefully 
choosing a measure for quantifying the quality of human-robot interactions.

Keywords: human-robot interaction; joint action; social simon effect.

\section{Introduction}

As robots are becoming more and more integrated in everyday life, recent research investigated the parameters characterizing interactions between humans and robots. In these human-robot interaction studies mostly questionnaires have been used to study different aspects of the interaction quality, including conscious measures of how robots are perceived (e.g. regarding their animacy and intelligence ${ }^{1}$ or regarding the empathy participants feel for a robot $^{2}$ ). Besides using questionnaires in humanrobot interaction studies, in the last years some studies used paradigms adapted from cognitive psychology, like motor interference paradigms ${ }^{3}$ or motion-capture technique $^{4}$, as indirect, behavioral indicators to study reactions towards a robotic agent. In the present study we applied the social Simon task in a joint action setting between a human and a robot. The social Simon task is an indirect, non-conscious behavioral measure commonly used to study to what extent humans co-represent the actions of other human interaction partners when acting jointly, a phenomenon referred to as action co-representation. In a broader sense, the social Simon Task has been interpreted to measure the degree to which people integrate the other person in the self ${ }^{5}{ }^{6}$. For interactions between humans, action co-representation has been shown to depend on the interpersonal relationship between two humans, with stronger co-representation effects the closer the relationship. Here, we aimed to test if the amount of co-representation of robotic actions correlates positively with the consciously perceived integration of a robotic agent in the self. To investigate this question, we reanalyzed data from a recent study of our labs ${ }^{7}$ with respect to this question, adding new conscious rating measures of self-other integration. While human participants showed evidence for self-other integration of a robotic agent in an indirect and unconscious test, evidence for self-other integration as obtained with a more conscious measurement was, however, weak. Conscious and unconscious measurements of self other integration may be dissociated from each other in human-robot interaction.

\subsection{The social Simon task}

The social Simon task, which was developed by Sebanz et al. in $2003^{8}$, is a well established paradigm from cognitive psychology for measuring relatively automatic joint-action effects. It has been mainly applied to human-human interactions. The social Simon task is based on the standard Simon task, which was first developed by Simon and Rudell in $1967^{9}$. In the standard Simon task one of two stimuli, e.g. a square or a diamond, is displayed on the left or right side of a monitor. A single person reacts to both stimuli by pressing one of two buttons, which are located to the left and right side of the monitor. For example, his or her task is to press 
the left button when a square appears on the screen, and to press the right button, when a diamond appears. The task relevant information is the shape of the stimulus. Although the spatial stimulus location is irrelevant for solving the task correctly response times are usually faster when stimulus location (left or right side of the screen) and response location (left or right button) correspond compared to when they do not correspond, as spatially corresponding stimuli facilitate responses, whereas spatially non-corresponding stimuli interfere with responses ${ }^{10}$. The difference in response times between corresponding and non-corresponding trials is called the Simon effect ${ }^{9} 1112$.

In a study of Sebanz et al. $(2003)^{8}$ two persons sitting side by side in front of a monitor shared a standard Simon task, so that each person was responsible for only one part of the task. This means that the left person pressed a button when one of two possible stimuli appeared on the screen, and the right person pressed a button when the other stimulus was displayed. When both persons performed their part of the task together (social Simon task or Joint Go/Nogo Task), a Simon effect was observed in each of them (social Simon effect, SSE). That is, their responses were faster, when the stimulus location corresponded with the location of their response button. No Simon effect was observed when one person performed his or her part of the task alone (Individual go/nogo task) ${ }^{8}{ }^{13}$, although in this Individual condition the sitting position of the participant and the location of the response button were the same as in the Joint condition. Even in an Individual condition, in which another person was passively sitting besides the participant, and not responding to the other part of the task, no Simon effect was observed ${ }^{8}$. On the other hand, when participant's are aware that another person is performing the other part of the task, not hearing the sounds of the partner's key presses or not seeing the partner (after being blindfolded) is not enough to eliminate the $\mathrm{SSE}^{814}$. Thus, the crucial factor for the SSE to occur is the active involvement of the other person in the task, whereas the SSE does not seem to rely on the perception of the action effects produced by the other person. These observations led Sebanz and colleagues to the conclusion that the SSE can be interpreted as an index of action co-representation ${ }^{8}{ }^{15}{ }^{16}$. That is, when acting together each participant automatically seems to integrate the actions of their partner into his own representation of the task, just as if each of them was in charge of the partner's action themselves. Co-representation may also contain information about whether and when the interaction partner has to respond ${ }^{17} 18$.

\subsection{Co-representation of non-biological agents}

Action co-representation was first believed to be restricted to human agents. Evidence for this assumption came from a study of Tsai and Brass $(2007)^{19}$, in which participants conducted a social Simon task either with a video showing the hand of a human agent or of a non-biological, wooden agent. A SSE was only observed when participants performed the task with the human agent. In another study by Tsai, Kuo, Hung, and Tzeng $(2008)^{20}$ participants performed a social Simon task while 
being told to share the task either with a computer or with another human sitting in another room (not visible to participants). Evidence for action co-representation was only found for those participants who believed to share the task with another human. From these findings Tsai and colleagues concluded that humans only corepresent the actions of other humans, and speculated that this might have evolved in order to facilitate interactions with conspecifics.

However, recent studies showed that action co-representation can occur for nonbiological agents depending on the degree of humanness attributed to the other agent. In a study of Müller and colleagues $(2011)^{21}$ participants performed a social Simon task with a wooden hand, but prior to the task, they either watched a short video sequence about a human actor or a wooden actor behaving in a human-like way (a sequence from the Walt Disney movie Pinocchio). Only those participants who had watched the video about the wooden agent, showed evidence for action co-representation when performing the social Simon task with the wooden hand. Müller and colleagues concluded that action co-representation can occur for nonbiological agents when perceiving an agent as animate. Regarding interactions with real robotic agents, a study from our labs showed that action co-representation is sensitive to the believed humanness of a robot's functional principle ${ }^{7}$. Prior to performing a social Simon Task together with a robot, participants were either told that the robot functioned in a human-like, intelligent way (human-like robot condition), or that it functioned in a machine-like, deterministic way (machine-like robot condition). Participants were randomly assigned to one of both conditions with the constraint that both groups were matched regarding gender. Participants who performed a social Simon task with the robot which they believed to function similar to a human, showed evidence for action co-representation. In contrast, those participants who were told that the robot functioned in a machine-like way did not co-represent the robot's actions. These studies suggest that higher order cognitive processes, like the belief about an agent's animacy or its functional principle, affect the amount of action co-representation in a top-down manner.

\subsection{Which aspects of the interpersonal relationship between two humans affect action co-representation?}

For human-human interaction it has been shown that the amount of action corepresentation is sensitive to the interpersonal relationship between two interacting individuals. For example, Hommel, Colzato, and van Wildenberg $(2009)^{22}$ found that action co-representation is stronger when both interaction partners were in a positive relationship. In their study, only participants who co-acted with a friendly, supporting co-actor, co-represented their partner's action, whereas participants who performed the task with an unfriendly, intimidating co-actor did not. In line with these results, Iani and colleagues $(2011)^{23}$ found evidence for action co-representation only when both participants cooperated in the social Simon task trying to achieve a common goal (the best performing couple received reward) but 
not when participants competed in the task (best performing participant received reward). Recent findings from Colzato et al. $(2012)^{5}$ suggest that the consciously perceived closeness towards others also determines to which extend his or her actions are co-represented. In their study, practicing buddhist, who learn by their religious practice to remove the barriers between themselves and others, showed stronger action co-representation effects than a matched control group of atheist. Colzato et al. concluded that the higher closeness buddhist feel towards their fellow beings results in a stronger integration of the other person in the self including the integration of the other persons actions. In summary, it can be stated that in human-human interactions the shared representational system is sensitive to the consciously perceived interpersonal relationship between two co-acting human individuals, with stronger co-representation effects for more closely perceived relationships.

But what about interactions between humans and robots? Does the consciously perceived self-other integration with a robot go hand in hand with a more indirectly and automatically measured co-representation of robotic actions? This question is highly important, because the major amount of research on human-robot interaction currently uses conscious questionnaire measurements. Humans do not only attach to other humans, but also to objects of great personal importance to them. This is not only true for children, who often attach emotionally to dolls or other toys ${ }^{24} 25$ but also for adults who are often emotionally attached to pieces of art or personal memorabilia ${ }^{26}$. Further, there seem to exist gender-specific differences regarding the type of objects that are attached to: For example, men more often name technical devices as an object of attachment than women ${ }^{27}$.

\subsection{Purpose of the present study}

In the present study we investigated whether co-representation of robotic actions (as a unconscious measure of self-other integration) is positively correlated with the perceived closeness felt towards a robot (a conscious measure of self-other integration). Participants performed a social Simon task together with a robot. After the task participants had to indicate the perceived closeness they felt toward the robot with the help of the Inclusion of the Other in the Self Scale (IOS scale) ${ }^{28} 29$, a measure taken from social psychology used to indicate the closeness and attraction in an interpersonal relationship between humans. The SSE was calculated by subtracting averaged response times (RTs) in spatially corresponding trials from averaged RTs in spatially non-corresponding trials. The size of the SSE, taken as an index of action co-representation, and the IOS-score were correlated first for the entire group of participants and second separately for male and female participants. Given the finding of Colzato et al. ${ }^{5}$ from human-human interaction research, we expected an overall positive correlation between conscious and unconscious measures of self-other integration. We also tested whether there exist gender-specific differences in the correlation. If men more easily attach emotionally to technical devices than women, one may expect such gender-specific differences regarding conscious 
and unconscious measures of self-other integration in human-robot interaction.

\section{Method}

The results of the correlation analyses between concious and unconcious measurements of self-other integration are the result of a new analyses of the data partially described in the study by Stenzel et al. ${ }^{7}$.

\subsection{Participants}

Our sample comprised 48 students from the University Jaume I (Castellon, Spain), among them were 24 female (mean age $=20.4$ years, range $=18$ to 38 years) and 24 male (mean age $=19.9$ years, range $=18$ to 24 years) participants. All participants were treated according to the ethical guidelines laid down in the 1975 Declaration of Helsinki. Each participant received 10 Euro for participation.

\subsection{Robot}

The robot Tombatossals which was devised and assembled in the robotic intelligence laboratory at the University of Castellon served as the partner in a social Simon task (Figure 1a). Tombatossals is a humanoid torso with a pan/tilt/vergence anthropomorphic head, eyes-cameras, arms, and a three-finger, four-degrees-of-freedom Barrett Hand (left hand) ${ }^{30}$.

A

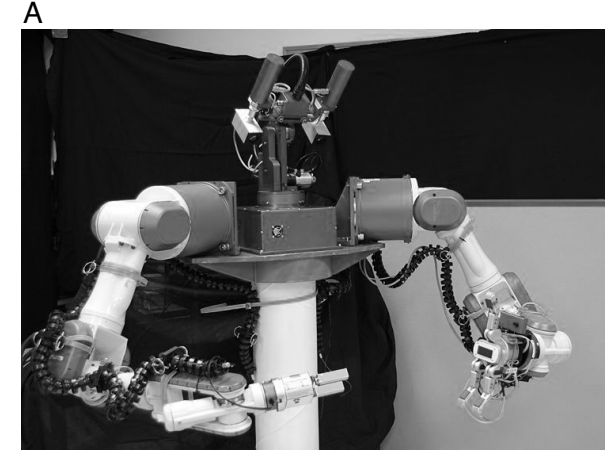

B

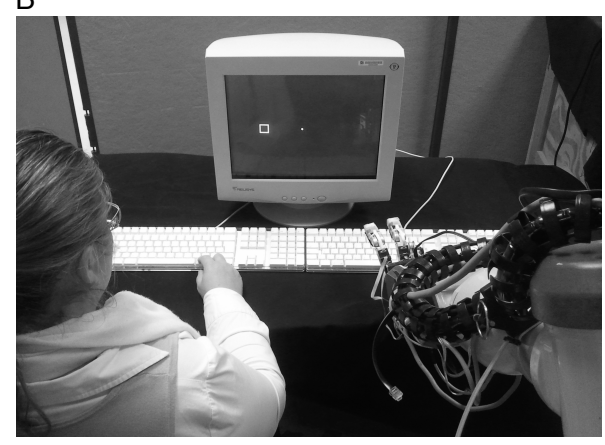

Fig. 1. Experimental setup used in the experiment. Participants performed a social Simon task together with a humanoid torso (see left panel, A). In a social Simon task a standard Simon task is distributed across two participants. In our study we used a square and a diamond as stimuli which were presented on the left or right side of the monitor. The participant reacted to the square and the robot reacted to the diamond by pressing a keyboard button (see right panel, B). 


\subsection{Apparatus, Stimuli and Procedure}

The experimental program ran on a MacBook Pro. We used a white square and a diamond $\left(1.9^{\circ} \times 1.9^{\circ}\right.$ of visual angle) as stimuli which were displayed on a 16 -in. CRT monitor with a viewing distance of about $60 \mathrm{~cm}$. As response button we used the left command key of two apple keyboards which were placed on a table next to each other (Figure 1b). At the beginning of each trial a white fixation point $\left(0.4^{\circ}\right.$ $\mathrm{x} 0.4^{\circ}$ of visual angle) was presented on a black screen for $250 \mathrm{~ms}$. During the next $250 \mathrm{~ms}$ either the square or the diamond appeared $8.0^{\circ}$ to either the left or right of the fixation point. Participants could give their response during the next 1800 ms. Afterwards, a visual feedback (duration: $300 \mathrm{~ms}$ ) informed them whether their response was correct or not: The fixation dot turned green after correct responses, and red after errors. Errors could either be misses (not pressing the button although the imperative stimulus was displayed) or false alarms (pressing the button although the imperative stimulus was not displayed). The next trial started after a constant inter-trial interval of $1850 \mathrm{~ms}$. Both stimulus types (square and diamond) were presented with equal probability (in $50 \%$ of trials on the left side, in the other $50 \%$ on the right side). The order of trials was pseudo randomized. All participants performed four blocks comprising 128 trials (512 trials in total). Between blocks they had a short break.

The participant always sat on the left side of the monitor (Figure 1b) and responded to the square by pressing the left response button with his or her right index finger. Participants were asked to respond as fast and as accurately as possible. The robot was located on the right side of the monitor (Figure 1b) and responded to the diamond by pressing the right response button with the rightmost finger of its left hand. When it was the robot's turn the two joints of the finger moved down simultaneously to press the button while the rest of the hand was kept completely still throughout the whole experiment. To make the robot's response times comparable to a human interaction partner, the robot was controlled by the experimenter who was sitting behind a panel. The experimenter wore headphones and heard a tone, every time the robot should give a response. When hearing the tone, the experimenter pressed a button to send a trigger signal to the robot, which initiated the finger movement. The robot's reaction times were comparable to a human interaction partner (mean reaction time: $371 \mathrm{~ms}$ ). Further, in each block two out of 128 trials ( $1.6 \%$ of trials) were error trials, so that the robot also made some errors, just like a typical human partner.

Although the robot actually functioned in a deterministic manner as it was controlled by an external person, we intended to make participants believe that the robot functions in a human-like way. Therefore, at the beginning of the experiment we provided participants with information about the robot portraying it either as an active and intelligent agent or a more passive agent (for details of the instruction $\left.\operatorname{see}^{7}\right)$. 


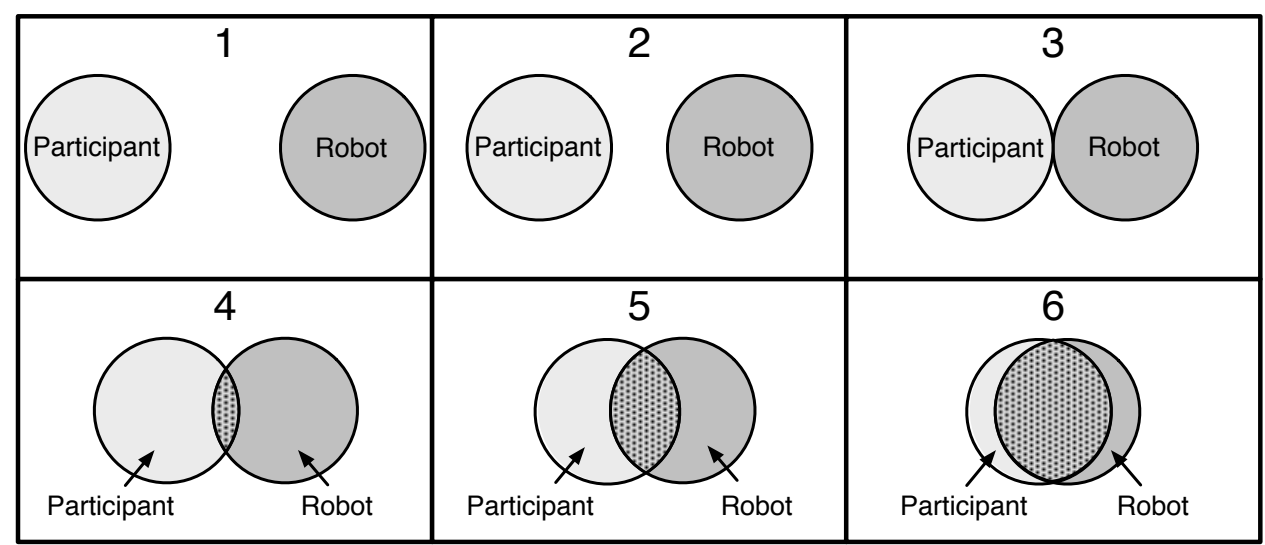

Fig. 2. Inclusion of the Other in the Self (IOS) scale. The IOS scale is a measure commonly used in social psychology to assess the emotional closeness and attraction in an interpersonal relationship. Participants were asked to indicate which of the six images best described the relationship between themselves and the robot. To illustrate the concept of the scale we added the expressions "Participant" and "Robot" to this image. In the scale used in our experiment these labels were not included.

\subsection{Inclusion of the Other in the Self scale}

To measure the consciously perceived closeness of the relationship between the participant and the robot, we used a modified version of the Inclusion of the Other in the Self Scale (IOS scale) developed by Aron and colleagues ${ }^{28}{ }^{29}$, which participants filled out after they had performed the social Simon task with the robot. The IOS scale was developed as a conscious measure of perceived closeness in intimate relationships, but it was also shown to reflect the perceived emotional closeness and attraction of a relationship created in a laboratory situation. Participants usually interpret this scale as a measure of the interconnectedness between themselves and another person ${ }^{29}$. In the IOS scale Aron and colleagues incorporated the idea of perceived closeness as overlapping selves. The IOS scale we used consisted of six pictures depicting different degrees of overlap of two circles ranging from completely separated (IOS score: 1) to strongly overlapped (IOS score: 6) (see Figure 2). A higher IOS score indicated higher perceived closeness to the robot. Participants were asked to choose the picture which best described the relationship between themselves and the robot. We did not provide participants with any further information about how to interpret the IOS scale. We expected, that the IOS scale would most likely be interpreted as indicating the attraction or fascination participants felt for the robot. 


\section{Results}

We first discarded all trials from the analysis in which the participant or the robot made an error. The participant's response on diamond trials was classified as an error (error type false alarm). As we were only interested in participant's RTs to the square, we therefore excluded these trials. Furthermore, as only the first response time in a trial was recorded, trials in which the robot reacted to the square (error type "false alarm" for the robot) were also excluded. One male participant had to be excluded from the analyses because he did not fill out the IOS scale.
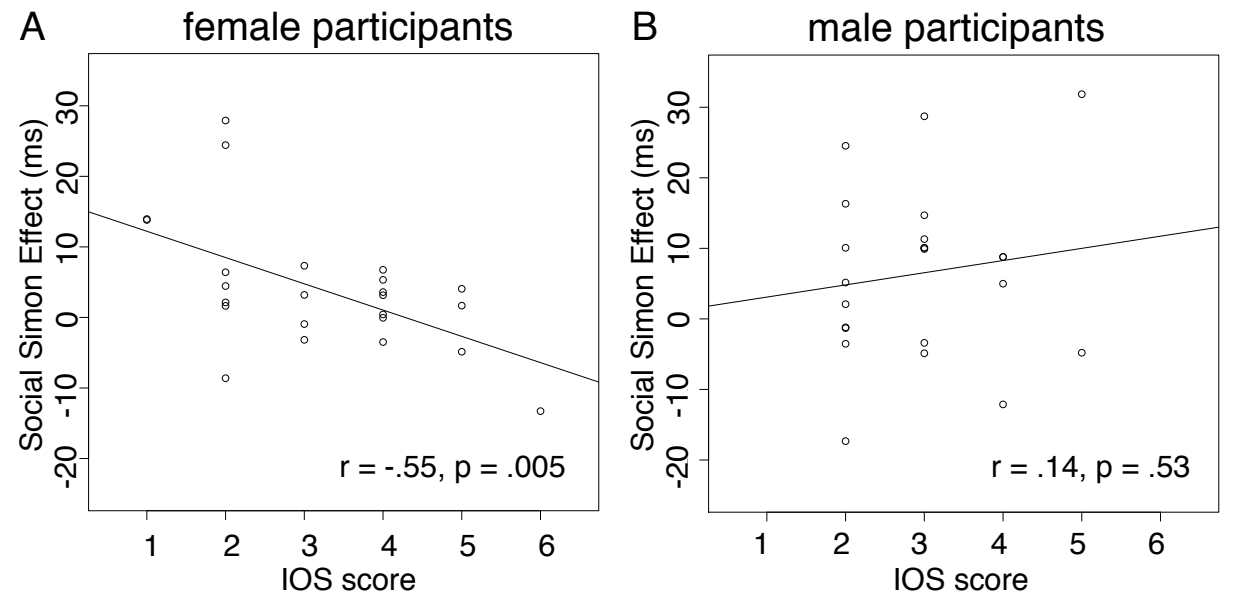

Fig. 3. Correlations between the size of the SSE and the IOS score plotted separately for female (left panel, A) and male (right panel, B) participants. r: Pearson's correlation coefficient, p: significance value.

For each participant we calculated median RTs for trials with correspondence and trials with non-correspondence between stimulus location and location of the response effector. The size of the SSE was calculated by subtracting RTs in corresponding trials from RTs in non-corresponding trials. We first investigated whether the SSEs for the whole group and for men and women separately were significantly different from zero, and investigated whether there exist gender differences in the mean values of the SSE and IOS by calculating two-sided t-tests. Further, we calculated correlations (Pearson's r) between the size of the SSE and the IOS score for all participants and separately for male and female participants. The size of the correlation and the two-tailed p-values are reported.

For the whole group, we observed a significant SSE $(5 \mathrm{~ms}, \mathrm{t}(46)=3.4, \mathrm{p}=.002)$ reflecting an unconscious effect of self-other integration. This effect was present in both male $(6 \mathrm{~ms}, \mathrm{t}(22)=2.56, \mathrm{p}=.02)$ and female $(4 \mathrm{~ms}, \mathrm{t}(23)=2.14, \mathrm{p}=.04)$ participants. There were no significant gender differences regarding the mean SSE, 
$\mathrm{t}(45)=.79, \mathrm{p}=.43$, nor did we find gender differences regarding the mean IOS score (mean male $=3.0$, mean female $=3.2, \mathrm{t}(45)=-.73, \mathrm{p}=.47)$. The overall mean IOS score of 3.1 corresponded to the picture of two circles which were arranged very close to each other, but did not overlap.

For the whole group, we found no significant correlation between the SSE and the IOS score, $\mathrm{r}=-.22, \mathrm{p}=.14$. Numerically we found a small trend for a negative correlation. For male participants this correlation was non-significant, $\mathrm{r}=.14 \mathrm{p}$ $=.53$. For female participants we observed a significant negative correlation between the size of the SSE and the IOS score, $\mathrm{r}=-.55, \mathrm{p}=.005$, indicating that the larger the SSE, the less did female participants consciously perceive the robot to be integrated in the self. The size of the correlation coefficient was significantly different between male and female participants, $\mathrm{z}=2.43, \mathrm{p}=.015$.

\section{Discussion}

The majority of research on human-robot interaction used conscious questionnaire measurements to investigate how robots are perceived regarding characteristics such as animacy or intelligence ${ }^{1}$. More recently, indirect and unconscious behavioral indicators were used for measuring the quality of human-robot interactions ${ }^{3}{ }^{4}$. In the present study, we investigated the relationship between an indirect, unconscious measurement of self-other integration (the $\mathrm{SSE}^{8}{ }^{15}$ ) and a conscious measure of self-other integration (the IOS scale ${ }^{28} 29$ ). We further investigated whether there exist gender specific differences regarding this correlation. Based on human-human interaction studies ${ }^{5}$ we expected to find a positive correlation between both measures. Over all participants, as well as for the group of male and the group of female participants, we found a significant SSE, i.e. evidence that participants integrated the robot's actions in their own task representation at an unconscious level. When consciously asked to rate self-other integration, both male and female participant's mean scores showed no evidence for the inclusion of the robot in the self. Further, we did not observe the predicted correlation between the unconscious and conscious measurements of self-other integration. While male participants showed absolutely no correlation, the correlation between SSE and IOS was even reversed for female participants. This pattern seems to be different from what is observed for human-human interaction studies showing a positive association between the SSE and the conscious perception of integrating others in the self ${ }^{5}$. Our findings seem to reveal a dissociation of unconscious and conscious measurements of self-other integration for human-robot interactions. While participants integrate the robotic agent in the self at an unconscious level, they do not report feelings of perceived closeness towards the robot at a more conscious level. These findings seem highly relevant for the technical and engineering side of robotics. When developing new robotic architectures, the only way to adequately test them may be to adopt indirect, more unconscious measures. Using explicit questionnaires may or may not reveal advances of the robotic system. The problem with explicit questionnaires may 
be due to introspection of human participants. Humans often do not have conscious access of the actually internally ongoing mechanisms. Even when they start to integrate the robotic agent in the self on an unconscious level, an explicit question on self-other integration may lead to an active inhibition of conscious self-other integration. In our study, this was particularly true for female participants: The larger their unconscious integration of the robotic agent was, the less did female participants consciously perceive the robot to be integrated in the self.

This points to the role of behavioral measures adopted from human-human interactions for measuring the interaction quality of human-robot interactions. It seems to be important that the measures used to index attitudes or emotional reactions towards a robot are given in a non-conscious way. Self-report measures might not always be the appropriate way to indicate attitudes towards a robot.

Another interesting finding concerns the lack of a difference in the overall mean IOS score between male and female participants. This finding is not in line with the fact that men more easily attach to technical devices than women ${ }^{27}$. However, our findings may suggest gender-specific differences with respect to the conscious evaluation of self-other integration of a robotic interaction partner.

An interesting aspect for future research might be to more directly test genderspecific differences of different levels of self-other integration between human-human and human-robot setups. Furthermore, future research should investigate whether the negative correlation pattern we observed for female participants may be reversed when choosing a robot as interaction partner that can be more easily emotionally attached to. A stronger emotional attachment might make it easier to consciously perceive a deeper form of emotional closeness felt for the robot. This may be achieved by making the robot more human-like, for example regarding its physical appearance (e.g. a robot with more human-like facial features) or its behavior (e.g. a robot with human-like communication skills).

\section{Conclusions}

The present study shows no overall correlation between unconscious and conscious measurements of self-other integration for human-robot interactions. While human participants seem to be open to integrate other human interaction partners in the self on a conscious and an unconscious level, they start to actively inhibit self-other integration for robotic partners when consciously asked, although they are open to unconscious integration. Our findings indicate that conscious and unconscious evaluations of a robotic agent might lead to different results, pointing to the importance of carefully choosing a measure for evaluating human-robot interactions.

\section{Acknowledgements}

This research was supported by the European Commission Project FP7-ICT217077-Eyeshots, the German Research Foundation project DFG LI 2115/1-1, and 
by WCU (World Class University) program through the National Research Foundation of Korea funded by the Ministry of Education, Science and Technology (Grant No. R31-2008-000-10062-0).

\section{References}

1. C. Bartneck, D. Kulic, E. Croft, and S. Zoghbi. Measurement instruments for the anthropomorphism, animacy, likeability, perceived intelligence, and perceived safety of robots. International Journal of Social Robotics, 1:71-81, 2009.

2. L. D. Riek, T.-C. Rabinowitch, B. Chakrabarti, and P. Robinson. How anthropomorphism affects empathy toward robots. In Proceedings of the 4 th ACM/IEEE international conference on Human robot interaction, HRI '09, pages 245-246, New York, NY, USA, 2009. ACM.

3. A. Kupferberg, S. Glasauer, M. Huber, M. Rickert, A. Knoll, and T. Brandt. Biological movement increases acceptance of humanoid robots as human partners in motor interaction. AI and Society, 26:339-345, 2011.

4. T. Kanda, H. Ishiguro, M. Imai, and T. Ono. Development and evaluation of interactive humanoid robots. Proceedings of the IEEE, 92(11):1839-1850, 2004.

5. L. Colzato, H. Zech, B. Hommel, R. Verdonschot, W. van den Wildenberg, and S. Hsieh. Loving-kindness brings loving-kindness: The impact of buddhism on cognitive self-other integration. Psychonomic Bulletin and Review, 19:541-545, 2012.

6. R. Liepelt, J. C. Schneider, D. Aichert, N. Wöstmann, S. Dehning, H. J. Möller, M. Riedel, T. Dolk, and U. Ettinger. Action blind: Disturbed self-other integration in schizophrenia. Neuropsychologia, 50(3775-3780), 2012.

7. A. Stenzel, E. Chinellato, M. A. Tirado Bou, A. P. del Pobil, M. Lappe, and R. Liepelt. When humanoid robots become human-like interaction partners: co-representation of robotic actions. Journal of Experimental Psychology: Human Perception and Performance, in press.

8. N. Sebanz, G. Knoblich, and W. Prinz. Representing others' actions: just like one's own? Cognition, 88(3):B11-B21, 2003.

9. J. R. Simon and A. P. Rudell. Auditory s-r compatibility: The effect of an irrelevant cue on information processing. Journal of Applied Psychology, 51(3):300-304, 1967.

10. S. Kornblum, T. Hasbroucq, and A. Osman. Dimensional overlap: Cognitive basis for stimulus-response compatibility - a model and taxonomy. Psychological Review, 97:253-270, 1990.

11. J. R. Simon. Reactions toward the source of stimulation. Journal of Experimental Psychology, 81(1):174-176, 1969.

12. J. R. Simon and A. M. Small. Processing auditory information: Interference from an irrelevant cue. Journal of Applied Psychology, 53(5):433-435, 1969.

13. R. Liepelt, D. Wenke, R. Fischer, and W. Prinz. Trial-to-trial sequential dependencies in a social and non-social simon task. Psychological Research, 75:366-375, 2011.

14. E. Vlainic, R. Liepelt, L. S. Colzato, W. Prinz, and B. Hommel. The virtual co-actor: the social simon effect does not rely on online feedback from the other. Frontiers in Psychology, 1, 2010.

15. N. Sebanz, G. Knoblich, and W. Prinz. How two share a task: Corepresenting stimulusresponse mappings. Journal of Experimental Psychology: Human Perception and Performance, 31(6):1234-1246, 2005.

16. D. Kiernan, M. Ray, and T. Welsh. Inverting the joint simon effect by intention. Psychonomic Bulletin and Review, pages $1-7$, in press.

17. T. Dolk, B. Hommel, L. S. Colzato, S. Schütz-Bosbach, W. Prinz, and R. Liepelt. 
How social is the social simon effect? Frontiers in Psychology, 2, 2011.

18. D. Wenke, S. Atmaca, A. Holländer, R. Liepelt, P. Baess, and W. Prinz. What is shared in joint action? issues of co-representation, response conflict, and agent identification. Review of Philosophy and Psychology, 2:147-172, 2011.

19. C.-C. Tsai and M. Brass. Does the human motor system simulate pinocchio's actions? Psychological Science, 18(12):1058-1062, 2007.

20. C.-C. Tsai, W.-J. Kuo, D. L. Hung, and O. J. L. Tzeng. Action co-representation is tuned to other humans. Journal of Cognitive Neuroscience, 20(11):2015-2024, 2008.

21. B. C. N. Müller, M. Brass, S. Kühn, C.-C. Tsai, W. Nieuwboer, A. Dijksterhuis, and R. B. van Baaren. When pinocchio acts like a human, a wooden hand becomes embodied. action co-representation for non-biological agents. Neuropsychologia, 49(5):13731377, 2011.

22. B. Hommel, L. S. Colzato, and W. P. M. van den Wildenberg. How social are task representations? Psychological Science, 20(7):794-798, 2009.

23. C. Iani, F. Anelli, R. Nicoletti, L. Arcuri, and S. Rubichi. The role of group membership on the modulation of joint action. Experimental Brain Research, 211:439-445, 2011.

24. E. Brauch Lehman, B. E. Arnold, and S. L. Reeves. Attachments to blankets, teddy bears, and other nonsocial objects: A child's perspective. The Journal of Genetic Psychology: Research and Theory on Human Development, 156(4):443-459, 1995.

25. B. M. Hood and P. Bloom. Children prefer certain individuals over perfect duplicates. Cognition, 106(1):455-462, 2008.

26. S. Schultz Kleine and S. Menzel Baker. An integrative review of material possession attachment. Academy of Marketing Science Review, 1:1-39, 2004.

27. M. Csikszentmihalyi and E. Rochberg-Halton. The Meaning of Things. London: Cambridge University Press, 1981.

28. A. Aron, E. N. Aron, M. Tudor, and G. Nelson. Close relationships as including other in the self. Journal of Personality and Social Psychology, 60(2):241-253, 1991.

29. A. Aron, E. N. Aron, and D. Smollan. Inclusion of other in the self scale and the structure of interpersonal closeness. Journal of Personality and Social Psychology, 63(4):596-612, 1992.

30. E. Chinellato, M. Antonelli, B. J. Grzyb, and A. P. del Pobil. Implicit sensorimotor mapping of the peripersonal space by gazing and reaching. IEEE Transactions on Autonomous Mental Development, 3(1):43-53, 2011. 